Instituto Internacional de Investigación y Desarrollo Tecnológico Educativo INDTEC, C.A. DOI: https://doi.org/10.29394/Scientific.issn.2542-2987.2020.5.16.3.62-81

OAI-PMH: http://www.indteca.com/ojs/index.php/Revista Scientific/oai

Artículo Original / Original Article

\title{
El conflicto escolar: una oportunidad para construir relaciones de convivencia con estudiantes de básica primaria
}

\author{
Autor: Edgar Alfredo Montaño Timana \\ Secretaría de Educación Municipal, SEMPASTO \\ emontano2000@hotmail.com \\ Pasto, Colombia \\ https://orcid.org/0000-0002-0515-3968
}

Resumen

Los conflictos, hasta hace algunos años estudiados desde una connotación negativa como producto de un problema, riña o agresión han ido paulatinamente cambiando y enmarcándose en un enfoque moderno y positivo, en donde especialmente en las organizaciones es asumido como algo natural e inevitable que se tiene que aceptar, manejar y solucionar sintetizando siempre los aspectos más positivos del mismo. El objetivo estuvo encaminado a analizar las oportunidades educativas que surgen del conflicto escolar y cómo éstas favorecen la construcción de relaciones de convivencia, ofreciendo un marco de reflexión para tratar constructivamente el conflicto escolar, modificando las prácticas cotidianas de asumirlo que impiden o afectan procesos saludables de crecimiento personal. El tipo de investigación es cualitativa, el cual favoreció descubrir, profundizar y enriquecer la comprensión de situaciones sociales relevantes en la realidad investigada buscando significados atribuidos por los propios protagonistas. Entre los resultados se encuentra que los estudiantes que han asumido significativamente sus conflictos crecen integralmente, mientras que quienes lo resuelven de manera disfuncional generalmente rompen relaciones y sufren afectaciones emocionales. Una de las conclusiones indica que las oportunidades educativas que surgen del conflicto escolar se establecen en correspondencia a la inteligencia emocional que desarrolla una persona.

Palabras clave: mediación; resolución de conflictos; convivencia pacífica; educación.

Cómo citar este artículo:

Montaño, E. (2020). El conflicto escolar: una oportunidad para construir relaciones de convivencia con estudiantes de básica primaria. Revista Scientific, 5(16), 62-81, e-ISSN: 2542-2987. Recuperado de: https://doi.org/10.29394/Scientific.issn.2542-2987.2020.5.16.3.62-81

Fecha de Recepción:

01-11-2019
Fecha de Aceptación:

24-02-2020
Fecha de Publicación:

05-05-2020 
OAI-PMH: http://www.indteca.com/ojs/index.php/Revista_Scientific/oai

Artículo Original / Original Article

\title{
The school conflict: an opportunity to build relationships with primary school students
}

\begin{abstract}
The conflicts, until a few years ago studied from a negative connotation as a product of a problem, quarrel or aggression have been gradually changing and framed in a modern and positive approach, where especially in organizations it is assumed as something natural and inevitable that has that accept, manage and solve always synthesizing the most positive aspects of it. The objective was to analyze the educational opportunities that arise from the school conflict and how they favor the construction of coexistence relationships, offering a framework for reflection to constructively address the school conflict, modifying the daily practices of assuming it that prevent or affect healthy processes of personal growth. The type of research is qualitative, which favored discovering, deepening and enriching the understanding of relevant social situations in the investigated reality, looking for meanings attributed by the protagonists themselves. Among the results is that students who have significantly assumed their conflicts grow integrally, while those who resolve it dysfunctionally generally break relationships and suffer emotional disturbances. One of the conclusions indicates that the educational opportunities that arise from the school conflict are established in correspondence to the emotional intelligence that a person develops.
\end{abstract}

Keywords: mediation; conflict resolution; peaceful coexistence; education.

How to cite this article:

Montaño, E. (2020). The school conflict: an opportunity to build relationships with primary school students. Revista Scientific, 5(16), 62-81, e-ISSN: 2542-2987. Recovered from: https://doi.org/10.29394/Scientific.issn.2542-2987.2020.5.16.3.62-81

Date Received:

01-11-2019
Date Acceptance:

24-02-2020
Date Publication: 05-05-2020 
OAI-PMH: http://www.indteca.com/ojs/index.php/Revista_Scientific/oai

Artículo Original / Original Article

\section{Introducción}

La temática planteada en este estudio, surge como una preocupación personal y profesional en el trabajo académico que a diario se realiza con niños de establecimientos educativos de básica primaria. Preocupación que se agudiza al ver situaciones como: estudiantes que con mucho temor, nerviosismo, irritación o antipatía pretenden salir avante en conflictos escolares; niños que le huyen al conflicto siendo complacientes y dejándose amilanar por sus pares; niños que asumen el conflicto pero con resultados disfuncionales rompiendo relaciones sociales con heridas emocionales desastrosas o niños que atribuyen significados distintos al conflicto, engrandeciéndolo y traduciéndolo a sinónimo de provocación con resultados adversos tanto para él como para el grupo interviniente. Los directivos por su parte aplicando las normas establecidas en el manual de convivencia pretenden disminuirle la fuerza a un conflicto y lo dan por terminado a la firma de un acta de compromiso.

Son situaciones que están retrocediendo la perspectiva funcional con la que debe asumirse el conflicto es decir se está abordando la aparición, desarrollo y desenlace de este, desde una perspectiva tradicional, planteando que el conflicto es dañino y debe evitarse a toda costa dejando a un lado el concepto enriquecedor que diversos autores exponen del tema y que incluso lo consideran como algo natural e ineludible en nuestra sociedad.

Es desde allí, de donde surge la necesidad de estudiar el conflicto escolar y su incidencia en las habilidades sociales y emocionales que se mueven al interior y exterior de cada persona. Su matiz positivo evoca en la comunidad educativa el aporte de nuevos conocimientos e ideas innovadoras como es el manejo de las emociones concebidas desde las primeras etapas de crecimiento las cuales precisan la personalidad del niño, expresiones, sentimientos y habilidades sociales. Correspondiendo de manera asertiva situaciones particulares que llevan a edificar en las personas el disfrute de una 
OAI-PMH: http://www.indteca.com/ojs/index.php/Revista_Scientific/oai

Artículo Original / Original Article

situación más ventajosa en todos los dominios de la vida. El conflicto escolar y las oportunidades educativas que este brinda, se observan como un tema de interés general poco abordado desde esta perspectiva, brindando además pistas para emprender nuevas investigaciones con grandes contribuciones al campo educativo en nuestro país.

El objetivo general formulado, se apoyó en analizar las oportunidades educativas que surgen del conflicto escolar y favorecen la construcción de relaciones de convivencia y paz en estudiantes de educación básica primaria; ofrece pistas al educador para implementar en la institución educativa una formación integral que apunte no solo a favorecer el desarrollo intelectual del niño sino también sus competencias emocionales, afectivas y sociales, encaminadas a instaurar entornos escolares y familiares más saludables.

\section{Metodología}

Esta investigación cualitativa, permitió conocer aspectos de una comunidad, ahondando en situaciones sociales relevantes a partir de la voz de sus propios actores, y a su vez, en el estudio de las oportunidades educativas que se muestran a raíz del conflicto escolar y de qué forma favorecen estas la construcción de relaciones de convivencia, en estudiantes de cuarto y quinto grado de educación básica primaria. Estuvo encauzado a ejecutar un análisis profundo de las vivencias y percepciones que los participantes tienen acerca del tema. En cuanto al verbo con el cual se formula el objetivo general, Schettini y Cortazzo (2015), anotan que encarar la tarea de analizar significa: "asumir posturas tanto académicas como ideológicas y es justamente ese posicionamiento el que nos enfrentará a los apasionantes retos de la riqueza analítica que nos llevará a lo más profundo del fenómeno estudiado" (pág. 7). La tarea de analizar demanda condensar la información obtenida, pensar en significados, interpretaciones y conclusiones, trazando el 
camino que sigue la investigación.

Las herramientas cualitativas que se usaron para la recolección de información consintieron que la comunidad participante exprese abiertamente sus opiniones y puntos de vista acerca del tema. Entre los instrumentos utilizados para la recolección de información se tuvo en cuenta: el cuestionario abierto, la observación sistemática y la revisión documental.

La investigación incluyó dentro de la población, tres (3) instituciones educativas ubicadas en la zona suroriental del Municipio de Pasto, Colombia, se tuvo en cuenta la población estudiantil de los grados cuarto y quinto de Básica primaria y padres de familia, durante el año lectivo 2014.

Tabla 1. Características de la muestra seleccionada.

\begin{tabular}{|c|c|}
\hline $\begin{array}{l}\text { Criterio de } \\
\text { experto }\end{array}$ & Características de la muestra seleccionada \\
\hline \multirow{11}{*}{ Investigador } & Zona de influencia. \\
\hline & $\begin{array}{l}\text { Estudiantes de educación básica primaria grados cuarto y quinto } \\
\text { de primaria. }\end{array}$ \\
\hline & Padres de familia de estudiantes de cuarto y quinto de primaria. \\
\hline & $\begin{array}{l}\text { Condiciones de género un determinado número de niñas y otro de } \\
\text { niños. }\end{array}$ \\
\hline & Docentes de los grados cuartos y quintos. \\
\hline & Niños interesados en la temática. \\
\hline & Niños conflictivos. \\
\hline & $\begin{array}{l}\text { Niños que ocupan los primeros puestos, están en puesto } \\
\text { intermedios o en los últimos puestos. }\end{array}$ \\
\hline & Padres de familia interesados en la investigación. \\
\hline & $\begin{array}{l}\text { Docentes motivados a participar en el diligenciamiento del } \\
\text { cuestionario. }\end{array}$ \\
\hline & Niños menores de 12 años. \\
\hline
\end{tabular}

Fuente: El Autor (2014).

Los procedimientos para obtener la muestra y determinar el tamaño adecuado de esta, se hizo a partir de una exploración del contexto a estudiar, identificando a través de la observación directa: intereses, experiencias, miedos u motivaciones que pueden dar indicios de lo rica o pobre que pueda 
OAI-PMH: http://www.indteca.com/ojs/index.php/Revista_Scientific/oai

Artículo Original / Original Article

resultar la información. De igual forma, el criterio de experto descrito en la tabla 1 , integra ciertas generalidades de estudiantes, padres de familia y docentes que hacen parte de la muestra seleccionada; rasgos importantes de la comunidad que enriquecen y garantizan confiabilidad en el estudio.

El análisis de la información, se hizo utilizando un procedimiento secuencial y sistemático que recorren una serie de etapas, que van desde la transcripción y codificación de los textos, hasta la elaboración de campos categoriales y su lectura inicial, entregando resultados contundentes de la información explorada.

\section{Discusión de resultados}

Cuando se hace referencia al conflicto suele pensarse en este como algo desagradable y que despierta rechazo en la sociedad. En el momento varios investigadores han empezado a modificar la percepción de estos conceptos encontrando en ellos la oportunidad para movilizar y transformar prácticas cotidianas que pueden inferir o afectar procesos saludables de relación social.

El conflicto que se vive en los espacios escolares mueve múltiples posibilidades de aprendizaje, algunas de ellas relacionadas con: autoconocimiento, autocontrol, automotivación, reconocimiento de las emociones ajenas, relaciones interpersonales y competencias emocionales, que el ser humano edifica y que la escuela como institución formadora las fortalece en todos sus procesos educativos. En el momento, la función educativa no solo requiere preparar al estudiante para una profesión, involucra enseñar el saber ser, un concepto íntimamente ligado con las actitudes, valores y emociones, en este orden de ideas, Bisquerra (2000), menciona que: "la educación promueve competencias emocionales como elemento esencial del desarrollo humano, con objeto de capacitarle para la vida y con la finalidad 
OAI-PMH: http://www.indteca.com/ojs/index.php/Revista_Scientific/oai

\section{Artículo Original / Original Article}

de aumentar el bienestar personal y social" (pág. 243). De este modo, la educación fundamentada en competencias emocionales puede ser vista como un proceso educativo continuo y permanente, que mejora el desarrollo integral del ser humano, no solo en su proceso de formación escolar sino durante su vida en diferentes campos de actuación.

En los seres humanos, el autoconocimiento emocional hace referencia al conocimiento de sus propias emociones. En cuanto a esto, el análisis efectuado a la información suministrada por los participantes de la investigación, revela que un número determinado de niños que ha vivido situaciones de conflicto han desarrollado la conciencia de sí mismo; identificando que están sintiendo y porque lo hacen en el preciso momento que coexisten con el conflicto; una toma de conciencia que los lleva a estar más atentos y repensar sus actuaciones. Por otra parte, la inseguridad que otros niños viven en situaciones similares, el no saber qué hacer, como actuar y la falta de confianza en sí mismo pauta la imposibilidad de reconocer sus puntos débiles, exhibiendo insuficiente inteligencia emocional a la hora de enfrentar un problema.

Por su parte, Goleman (1996a): conceptúa el autoconocimiento como "el conocimiento de las propias emociones, es decir, la capacidad de reconocer un sentimiento en el mismo momento en que aparece" (pág. 31). Esto es, tener noción de sí mismo conociendo sus cualidades y siendo reflexivo en situaciones inesperadas. Un número definido de estudiantes que participan de la investigación exhiben un autoconocimiento emocional muy preciso al reaccionar en forma positiva ante un conflicto, recapacitando, responsabilizándose y dialogando con sus pares. Son reacciones que se producen a raíz de las experiencias que viven a diario en los espacios educativos y en el hogar. Varios de los niños que han pasado por un conflicto siguen momento a momento las expresiones de sus sentimientos el cual los ayuda a tener un mayor autoconocimiento y autocontrol en nuevas vivencias 
OAI-PMH: http://www.indteca.com/ojs/index.php/Revista_Scientific/oai

\section{Artículo Original / Original Article}

conflictivas que se pueden presentar.

El autoconocimiento es en definitiva una oportunidad educativa que surge cuando el niño vive el conflicto y asume constructivamente diferentes posiciones que le permiten salir a flote en situaciones apremiantes. Gran parte del autoconocimiento emocional estimula a la persona a conocer sus fortalezas, debilidades y diferentes estados de ánimo a los que se puede llegar en diversas circunstancias junto con las consecuencias que estos pueden acarrear; es una inteligencia que en el caso de los niños los llevarían a tener éxito en sus relaciones interpersonales encaminándolos a disfrutar de competencias emocionales más provechosa dentro del grupo.

Un segundo elemento que se desprende de la investigación se relaciona con el autocontrol emocional que en el caso de este estudio se presenta por medio del reconocimiento de sus actuaciones. Estas pueden ser vistas desde dos perspectivas, las acciones negativas, como: agresividad, pelea y sentimientos de rencor; y las actuaciones positivas que están caracterizadas por el dialogo, la calma y la tranquilidad.

El autocontrol emocional que exterioriza un determinado grupo de estudiantes en la investigación, se exhibe a la hora de explorar el tipo de actuaciones y los momentos de crisis que están viviendo, ajustando sus sentimientos a la situación, sin dejarse dominar por estos, reconociendo que un conflicto ofrece ambientes que pueden ser tensos pero que dependiendo de su autocontrol estos pueden llegar a ser momentáneos o pasajeros. Ante esto, Davidson (2012), destaca que:

Las personas (y los niños, concretamente) pueden aprender a disipar estas emociones negativas para volver al estado inicial. Es algo muy, muy importante, porque cuando las emociones perturbadoras se producen en la mente, interfieren con la capacidad de aprender de los niños (pág. 1-2).

Por consiguiente, la habilidad de controlar las emociones, es crucial 
para ayudar a que los niños aprendan mejor. Varios estudiantes aceptan haber vivido situaciones de conflicto con sus pares, sin embargo, son conscientes de haber asumido una posición tolerante, no dejándose envolver por el calor del momento que, implicaría actuar irresponsablemente engrandeciendo el conflicto, por el contrario, su actuación serena les ha permitido desenvolverse apropiadamente en dicho contexto. En relación con esto, Goleman (1996b), define que:

El autocontrol emocional es la conciencia de uno mismo, es una habilidad básica que nos permite controlar nuestros sentimientos y adecuarlos al momento, es la capacidad de tranquilizarse a uno mismo, de desembarazarse de la ansiedad, de la tristeza, de la irritabilidad exageradas y de las consecuencias que acarrea su ausencia (pág. 31).

El autor expone, que personas que carecen de esta habilidad, tienen que enfrentarse continuamente con las tensiones desagradables que una situación puede generar mientras que, por el contrario, las personas que la dominan reconocen, que es pasajero en una crisis y que perdura. En definitiva, la falta de autocontrol emocional en la persona impide regular los comportamientos volviéndolo impulsivo, sin tener oportunidad de detenerse a pensar o reflexionar las consecuencias de sus acciones. Se puede tener en cuenta, que niños de cuarto y quinto grado que durante el conflicto reaccionan con agresiones verbales, físicas y psicológicas, no tienen la capacidad para proceder acertadamente; reaccionan imponiendo comportamientos inapropiados. En consecuencia, su intervención en el conflicto es de forma destructiva o disfuncional.

La automotivación, surge a partir del deseo que tiene el niño de sacar a flote muchos de sus ideales, esta brota cuando hay objetivos a corto plazo que invitan a los estudiantes a gestionar recursos cognitivos, afectivos y psicomotores en busca de alcanzar las metas deseadas. En esa misma línea, Shapiro (1997), añade que: "Ios niños automotivados esperan tener éxito y no 
OAI-PMH: http://www.indteca.com/ojs/index.php/Revista_Scientific/oai

Artículo Original / Original Article

tienen inconvenientes en fijarse metas elevadas para sí mismos. Los niños que carecen de automotivación sólo esperan un éxito limitado" (pág. 129). La automotivación puede describirse como el motor interior que moviliza a la persona a conseguir sus ideales cultivando persistentemente el apetito de superación personal.

En el plano educativo, Bueno (1993), indica que:

La motivación para aprender hace referencia a una disposición continuada de valorar el aprendizaje como una actividad satisfactoria y merecedora de esfuerzo, de esfuerzo para conocer y dominar las situaciones de aprendizaje. Este rasgo es más característico en los sujetos que encuentran el aprendizaje intrínsecamente valioso (que se divierten 0 satisfacen aumentando sus conocimientos, incrementando su entendimiento de conceptos o procesos, o dominando destrezas) (pág. 36).

La automotivación promueve la búsqueda de unos objetivos, invitando a la persona a asumir nuevos comportamientos y posturas como hijos y como estudiantes que toleran resolver conflictos interpersonales al mismo tiempo, ser un ejemplo para otros y ser cada día mejor en diferentes escenarios de la vida.

Varios de los estudiantes que toman parte del estudio registran una progresiva automotivación que los lleva a plantearse objetivos a corto y mediano plazo, igualmente en forma permanente durante su vida escolar en los cuales se resalta como prioridad aprender, estudiar, mantener buenas relaciones, ser responsables, respetuosos y dialogar. Al respecto, Brophy (1983): expresa que los "estudiantes que están motivados no necesariamente encuentran las tareas de la clase intensamente placenteras o excitantes, sino que podrían hacer frente a ellas seriamente, encontrándolas significativas e intentar obtener el beneficio prefijado de ellas" (pág. 2). De allí que infundir esta competencia en las aulas es prioridad para el docente que inquiere 
OAI-PMH: http://www.indteca.com/ojs/index.php/Revista_Scientific/oai

\section{Artículo Original / Original Article}

mejores resultados en sus prácticas pedagógicas. En referencia al tema, Roca (2006), complementa que: "hablamos de automotivación cuando uno mismo planea regular la fuerza que le empuja a actuar, a partir del conocimiento que tiene sobre sí mismo" (pág. 10). La automotivación, es un impulso interno que proyecta en el presente, una línea de trabajo enfocada en metas alcanzables durante un periodo de tiempo.

Las personas que tienen esta capacidad, habitualmente desarrollan con mayor eficiencia sus actividades en las diferentes organizaciones que participan, en realidad personas que desde su infancia definen su proyecto de vida generalmente, son más provechosas en sus realizaciones tanto académicas como personales. Niños que direccionan sus emociones a un objetivo les permite estar atentos a las metas y no a los obstáculos que pueden entorpecer sus realizaciones. En varios estudiantes, la automotivación nace en diálogos con sus padres, consejos de sus profesores, familiares, directivos, orientador escolar y proyectos que se trabajan en el aula escolar como, el proyecto de vida y el de educación sexual. Son aspectos que motivan al estudiantado a plantearse un futuro exitoso sabiendo que quieren realmente.

El reconocimiento de las emociones ajenas se presenta en dos categorías que son: reconocer $y$, comprender lo que otros sienten situándose en el lugar de ellos. El primer elemento indica la capacidad de sintonizar adecuadamente las pequeñas señales que otros emiten dependiendo de su estado de ánimo; el segundo, comprender la situación emocional que otras personas están experimentando esto es, ponerse en los zapatos de ellos sintonizando sus emociones, entendiéndolas y respetándolas.

Referente a eso, Goleman (1996c), nombra al reconocimiento de las emociones ajenas como empatía y la conceptúa enunciando que:

Es el saber interpretar las señales que otras personas emiten en forma verbal como una mala contestación, una mala palabra igualmente en forma no verbal a través de señales con el cuerpo y gestos. El autor manifiesta que una persona empática 
OAI-PMH: http://www.indteca.com/ojs/index.php/Revista_Scientific/oai

Artículo Original / Original Article

capta las señales sociales más ligeras que revelan que pretenden los demás, esta capacidad hace a las personas más aptas para el desempeño de vocaciones (pág. 32).

Los estudiantes en su vida escolar ven la empatía como una oportunidad educativa que les ayuda a captar e interpretar los mensajes que sus compañeros emiten por medio del lenguaje verbal y también no verbal. Varios de los estudiantes reconocen diferenciar un ambiente hostil, discriminatorio y conflictivo al igual que los gestos improcedentes que emiten sus compañeros en el grupo. De allí que, actuaciones usuales como el no tenerlo en cuenta para integrar el equipo de deportes, no ser incluido en un grupo de trabajo y ser recriminado por otros, tocan particularmente las emociones de los niños perturbando su integración social, aprendizaje y permanencia en la escuela. En cuanto a este tipo de expresiones verbales y no verbales, a juzgar por Caballo (2007):

Es el medio por el que la gente se comunica con los demás y constituyen ambos los elementos básicos de la habilidad social. La comunicación no verbal es inevitable en presencia de otras personas. Un individuo puede decidir no hablar, o ser incapaz de comunicarse verbalmente, pero todavía sigue emitiendo mensajes sobre sí mismo a los demás por medio de su cara y su cuerpo. Los mensajes no verbales a menudo son recibidos de forma no consciente (pág. 24).

El estudiante empático identifica el estado de ánimo de uno o varios de sus pares, se ponen en su lugar, descubren lo que están sintiendo y pueden estar pensando, captan una gran cantidad de mensajes de sus compañeros a partir de sus palabras, así como interpreta mensajes que se transmiten en el lenguaje no verbal, por ejemplo: el tono de voz, movimientos corporales y las expresiones del rostro.

Con respecto a las relaciones interpersonales, estas se pueden dar de dos formas, la primera consiste en relacionarse e interactuar con las personas 
OAI-PMH: http://www.indteca.com/ojs/index.php/Revista_Scientific/oai

Artículo Original / Original Article

de la escuela, por ejemplo, entre estudiantes, docentes, administrativos y personal de servicios, la segunda, está en relacionarse e interactuar con otras personas en espacios diferentes al de la escuela, tales como la calle, un parque, un centro comercial u otros.

El manejo apropiado de las relaciones interpersonales que se viven en el medio escolar permite interactuar de forma reciproca entre dos o más personas, relaciones que además están sujetas a las normas de convivencia que se han implementado en la institución educativa. Dichas normas de convivencia hacen que el estudiantado asuma un comportamiento propicio para convivir en grupo y en sociedad. Algunos de esos comportamientos se vislumbran al momento de dirigirse a sus compañeros y profesores, por ejemplo: levantar la mano para opinar y expresarse adecuadamente dependiendo de las situaciones que se viven en el aula escolar y descanso; en los momentos de interacción social que surgen en encuentros culturales, deportivos y religiosos donde toda la comunidad educativa se congrega.

En correspondencia a las relaciones interpersonales, Goleman (1996d), afirma que:

El arte de las relaciones se basa, en buena medida, en la habilidad para relacionarnos adecuadamente con las emociones ajenas, estas son las habilidades que subyacen a la popularidad, el liderazgo y la eficacia interpersonal. Las personas que sobresalen en este tipo de habilidades suelen ser auténticas estrellas que tienen éxito en todas las actividades vinculadas a la relación interpersonal (pág. 32).

Las relaciones interpersonales hacen parte integral de la persona, por medio de ellas el ser humano logra contacto social con su entorno, grupo de trabajo, familia y demás personas, son ambientes que favorecen fundamentalmente su interacción social. Acorde con esto, Monjas (2004), explica que: "las habilidades sociales son un numeroso y variado conjunto de conductas que se ponen en juego en situaciones de interacción social, es 
OAI-PMH: http://www.indteca.com/ojs/index.php/Revista_Scientific/oai

Artículo Original / Original Article

decir, en situaciones en las que hay que relacionarse con otra/s persona/s" (pág. 12). La falta de manejo y control de las relaciones interpersonales provoca aislamiento y rechazo actitudes que restringen la interacción con otros y de hecho coarta su vida en sociedad. En este sentido, Delgado (2008), asegura que:

Las dificultades de relación social en la infancia se vinculan con problemas escolares (bajo rendimiento, absentismo escolar, abandono prematuro del sistema educativo); problemas externalizados en la adolescencia, como conducta antisocial y delincuencia en el caso de aquellos niños que han sido rechazados en la infancia por su conducta agresiva y disruptiva; y problemas internalizados durante el ciclo vital, incluyendo baja autoestima, problemas de ansiedad, soledad, y síntomas depresivos en aquellos niños caracterizados por un patrón de retraimiento y ansiedad en los años escolares (pág. 48).

Asimismo, crear y mantener contactos con diferentes personas, que tienen diferentes comportamientos y personalidades es una tarea diaria, e ineludible en la interacción social de los seres humanos. Cabe mencionar que, las habilidades sociales no son iguales en todos los individuos pues diversos factores influyen en ese aspecto, de allí la necesidad de fortalecer desde la niñez hasta la etapa adulta las relaciones entre pares que nacen en diversos escenarios como: el hogar, la calle y en especial la escuela, como así lo ratifica, Cadenas (2017), resaltando que: "hay que prestarle atención a la escuela porque es el espacio social y privilegiado para fomentar el pensamiento, el comportamiento valorativo, y para iniciar a practicar la ciudadanía a través de la convivencia" (pág. 357).

Es preciso registrar que las habilidades sociales como cualquier comportamiento puede regularse de manera positiva en las personas, adicionalmente Monjas y González (1998), subrayan que: "las habilidades sociales no mejoran por la simple observación ni por la instrucción informal; se 
necesita una instrucción directa... no se adquieren si no se llevan a cabo actividades educativas de forma intencional" (pág. 47). Debe anotarse que algunos estudiantes transforman fácilmente un comportamiento disfuncional a otro funcional, así como otros se resisten por temor, miedo al cambio o hacer tildados por sus compañeros. Son casos que requieren el acompañamiento del profesional del área que regule y oriente dichas emociones y provoque cambios en sus comportamientos, finalmente, edificándolos en habilidades sociales plausibles para vivir en comunidad.

\section{Conclusiones}

En función de los objetivos y preguntas problematizadoras propuestas en el trabajo de investigación y tras el análisis de los resultados obtenidos se concluye que:

Las oportunidades educativas que surgen del conflicto escolar se trazan en correlación a la inteligencia emocional que desarrolla una persona desde su infancia. Las personas con capacidad de conocer sus propios sentimientos y los de otras personas gobiernan significativamente sus emociones, tienen un autoconocimiento emocional, autocontrol emocional, automotivación, empatía y relaciones interpersonales más efectivas llevándolos a disfrutar de manera significativa y constructiva sus emociones.

El autoconocimiento emocional permite en los estudiantes destacar sus virtudes, reconocer puntos débiles y fuertes. Este autoconocimiento emocional propio en determinados niños de las instituciones investigadas, los lleva a confesar que sienten, a expresar abiertamente sus sentimientos, a reconocer los sentimientos de otros y a ponerse en el lugar del otro. Esa capacidad es la que crea sensibilidad en las personas dándole la oportunidad de actuar e intervenir en un conflicto midiendo sus actuaciones y alcances sin que estas afecten a otros emocionalmente.

El autocontrol emocional permite que los niños en el momento de un 
OAI-PMH: http://www.indteca.com/ojs/index.php/Revista_Scientific/oai

Artículo Original / Original Article

conflicto reconozcan sus actuaciones ante una crisis, aun cuando esta sea pasajera o perdure. Que los estudiantes conozcan la correspondencia que existe entre sus conductas y las emociones que pueden suscitar, es uno de los pasos más importantes en la comprensión del conflicto, generándose un aprendizaje en donde el estudiante confronta la forma de regular sus emociones para expresarlas de manera efectiva sin que dañen a otros o a ellos mismos. Los niños pueden ser educados para tener un autocontrol en situaciones de conflicto dándose una pausa para pensar, reflexionar y posteriormente actuar priorizando opciones que admitan gestionar el conflicto siempre en el marco del diálogo y el respeto.

La automotivación permite a los niños direccionar sus emociones hacia un objetivo manteniendo su atención en las metas y no en los posibles inconvenientes. Una buena automotivación permite a estudiantes descubrir la forma de superar inconvenientes presentados en su vida diaria. La automotivación como tercer elemento de la inteligencia emocional crea en el estudiantado iniciativa, responsabilidad, capacidad de salir avante en sus problemas, convirtiéndolo en un líder dentro del grupo. Esta inteligencia debería estar presente de manera transversal en los currículos educativos de todas las instituciones educativas del país fortaleciendo en los estudiantes su autoestima y proyecto de vida.

El reconocimiento de las emociones ajenas llamada empatía, se convierte en una oportunidad de aprendizaje cuando el estudiantado aprecia el sentir, la forma de ser y actuar de sus pares, para ello los estudiantes desarrollan la capacidad de entender los mensajes que otros transmiten no solo de manera verbal sino a través de sus gestos, tono de voz, movimientos corporales, forma de actuar, pensar y hasta sentir. La persona con esta inteligencia emocional desarrolla competencias afectivas, cognitivas y procesuales pues da respuestas afectivas y efectivas a la situación emocional 
que presentan sus pares, incentiva el pensamiento creativo poniendo activos todos sus sentidos y suscita situaciones en las que se desarrollan escenarios de apoyo y cooperación a compañeros que pasan por situaciones de crisis, problemas o conflictos. La persona empática tiene la habilidad emocional de ver los problemas desde afuera, comprenderlos, expresar sus opiniones sin dejarse llevar o involucrar por ellos, la empatía implica tolerancia un valor muy desprovisto en muchos padres de familia, estudiantes e incluso algunos docentes.

Las relaciones interpersonales en un conflicto escolar son las que se ponen en juego, pues este fragmenta lazos de unión que niños y niñas han construido por varios años. Estas relaciones interpersonales se convierten en una oportunidad educativa cuando el grupo de estudiantes invierte el conflicto y lo pone a su favor no destruyendo sino fortaleciendo sus relaciones dentro y fuera del sistema educativo, tratando a otros con respeto, estimulando la capacidad de trabajar en equipo e interactuando con ellos para encontrar respuestas y solucionar conflictos. Una buena relación con el grupo, los docentes, directivos y los padres de familia es fundamental en la vida de cada estudiante. Esas relaciones van más allá de saber tratar no solo con sus amigos, sino también con quienes se ha vivido situaciones de conflicto.

\section{Recomendaciones}

Resulta prioritario además de la enseñanza y aprendizaje de contenidos intelectuales, considerar en el currículo institucional y de forma transversal el aprendizaje de la inteligencia emocional. Una formación en donde se empiece a alfabetizar al estudiantado en competencias básicas para el manejo y fortaleciendo de su autoestima, proyecto de vida, trabajo empático, trabajo en equipo, resolución de conflictos, relaciones interpersonales y automotivación. De esta forma se estaría educando al niño en la forma de armonizar sus emociones ante diversas situaciones que se presentan en la vida. 
OAI-PMH: http://www.indteca.com/ojs/index.php/Revista_Scientific/oai

Artículo Original / Original Article

En las instituciones educativas que hacen parte de la investigación se visualiza ausencia de programas formativos dirigidos a potenciar las habilidades efectivas, emocionales y sociales en los docentes, que les ayuden a enfrentar y orientar situaciones emocionales que vive el estudiantado a diario en las aulas escolares y fuera de ellas. Es recomendable implementar planes formativos teórico-prácticos con profesores en el manejo de la inteligencia emocional en donde se desarrolle competencias educativas para conocer, valorar, controlar, mejorar las emociones y sentimientos personales y de los demás.

\section{Referencias}

Bisquerra, R. (2000). La educación emocional en la formación del profesorado. Barcelona, España: Editorial Wolters Kluwer.

Brophy, J. (1983). Conceptualizing student motivation. Occasional Paper, nro. 70. Michigan, EE.UU.: The Institute for Research on Teaching; Editorial Staff. Recovered from:

https://education.msu.edu/irt/PDFs/OccasionalPapers/op070.pdf

Bueno, J. (1993). La motivación en los alumnos de bajo rendimiento académico: desarrollo y programas de intervención. Tesis, Depósito Legal: M-30781-1993. Madrid, España: Universidad Complutense de Madrid. Recuperado de:

http://biblioteca.ucm.es/tesis/19911996/S/5/S5000201.pdf

Caballo, V. (2007). Manual de evaluación y entrenamiento de habilidades. España: Siglo XXI Editores.

Cadenas, Y. (2017). Capacitación en Valores para Fortalecer la Convivencia Social y Ciudadana. Revista Scientific, 2(3), 343-360, eISSN: 2542-2987. Recuperado de:

https://doi.org/10.29394/scientific.issn.2542-2987.2017.2.3.19.343-360 
Davidson, R. (2012). Aprender a gestionar las emociones. Emisión 130, temporada 17, Congreso del Mind \& Life Institutedel Mind \& Life Institute. Washington, Estados Unidos: Grupo Punset Producciones, S.A. Recuperado de: https://docplayer.es/13032271-Aprender-agestionar-las-emociones.html

Delgado, B. (2008). Psicología del desarrollo: desde la infancia a la vejez. ISBN: 978-84-481-6871-1. España: Editorial McGraw-Hill.

Goleman, D. (1996a,b,c,d). Inteligencia emocional. Barcelona, España: Editorial Kaíros.

Monjas, I. (2004). Ni sumisas ni dominantes. Los estilos de relación interpersonal en la infancia y en la adolescencia. Memoria de investigación. NIPO.: 207-05-059-2. España: Ministerio de Trabajo y Asuntos Sociales.

Monjas, I., \& González, B. (1998). Las habilidades sociales en el currículo. NIPO: 176-00-147-3; ISBN: 84-369-3414-8. España: Centro de Investigación y Documentación Educativa (CIDE) del Ministerio de Educación, Cultura y Deporte. Recuperado de: https://core.ac.uk/download/pdf/41588698.pdf

Roca, J. (2006). Automotivación. España: Editorial Paidotribo.

Schettini, P., \& Cortazzo, I. (2015). Análisis de datos cualitativos en la investigación social: Procedimientos y herramientas para la interpretación de información cualitativa. Argentina: Editorial de la Universidad de la Plata - EDULP. Recuperado de:

https://libros.unlp.edu.ar/index.php/unlp/catalog/download/451/416/149 $\underline{7-1}$

Shapiro, L. (1997). La inteligencia emocional de los niños. México: Javier Vergara Editor, S.A. 
OAI-PMH: http://www.indteca.com/ojs/index.php/Revista_Scientific/oai

Artículo Original / Original Article

\section{Edgar Alfredo Montaño Timana}

e-mail: emontano2000@hotmail.com

Nacido en Tangua, departamento de Nariño, Colombia, el

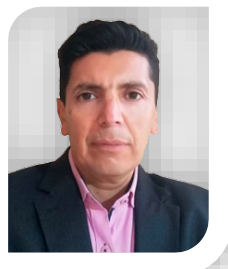
20 de marzo del año 1973. Licenciado en comercio y contaduría, Universidad Mariana (UNIMAR), Pasto; Especialista en multimedia educativa, Universidad Antonio Nariño (UAN), Pasto; Magister en docencia, Universidad de la Salle (UNISALLE), Pasto; Doctor en educación, Universidad de Baja California (UBC), México; Experiencia docente, Universidad Santo Tomás (UST), Facultad de Ciencias y Tecnologías del Centro de atención Universitario de Pasto; docente en varias instituciones educativas del departamento Nariño y municipio de Ipiales; En el momento, docente en propiedad de la Secretaria de Educación del Municipio de Pasto, Colombia desde el año 2010.

El contenido de este manuscrito se difunde bajo una Licencia de Creative Commons ReconocimientoNoComercial-Compartirlgual 4.0 Internacional 